\title{
THE HETEROGENEITY OF MUSCLE
}

by

\author{
OLIVER H. LOWRY
}

Department of Pharmacology, Washington University Medical School, 660 South Euclid Avenue, St. Louis, Missouri 63110 U.S.A.

Keywords: Individual muscle fibers, metabolite changes with intense stimulation, chronic stimulation effects

The basic principles of quantitative histochemistry, originated by HOLTER and LINDERSTRøM-LANG have been applied to skeletal muscle in several laboratories. Data are presented illustrating the remarkable differences in enzyme composition that exist among individual fibers in the same muscle. It is shown that enzyme values tend to separate somewhat into two groups, corresponding to the two major myosin ATPase staining types, but that otherwise the activities range as much as 10 -fold over an almost continuous spectrum. Other examples given at the individual fiber level concern (1) the affects of training and detraining on enzymes of energy metabolism, (2) the profound changes that can be produced by prolonged continuous motor nerve stimulation and (3) the striking differences in metabolite responses in different fiber types during intense stimulation.

\section{INTRODUCTION}

It has long been known that skeletal muscle fibers, even in the same muscle, differ markedly in regard to their twitch characteristics. Some are slow twitch with long endurance; some are fast twitch but fatigue rapidly; other are fast twitch but less fatigable.

In experimental animals there are individual muscles in which one or another of these three fiber types predominates. Analyses of such muscles has made it clear that the differences in twitch characteristics are associated with major

Abbreviations: $\mathbf{L D H}=$ lactate dehydrogenase; $\mathrm{MDH}$ = malate dehydrogenase differences in chemical composition. The slow twitch ("Type I") fibers are rich in enzymes of oxidative metabolism, but poor in enzymes of glycogenolysis. The fast twitch ("Type IIB") fibers are just the opposite: rich in enzymes of glycogenolysis (to supply quick ATP generation), but poor in oxidative enzymes (explaining the rapid fatigue). The intermediate ("Type IIA") fibers have all purpose enzyme characteristics: enzymes of glycogenolysis at intermediate levels and oxidative enzymes at levels which in rodents (but not in man) exceed those of Type I fibers.

In man, the muscles available for biopsy are an intimate mixture of all fiber types. This 


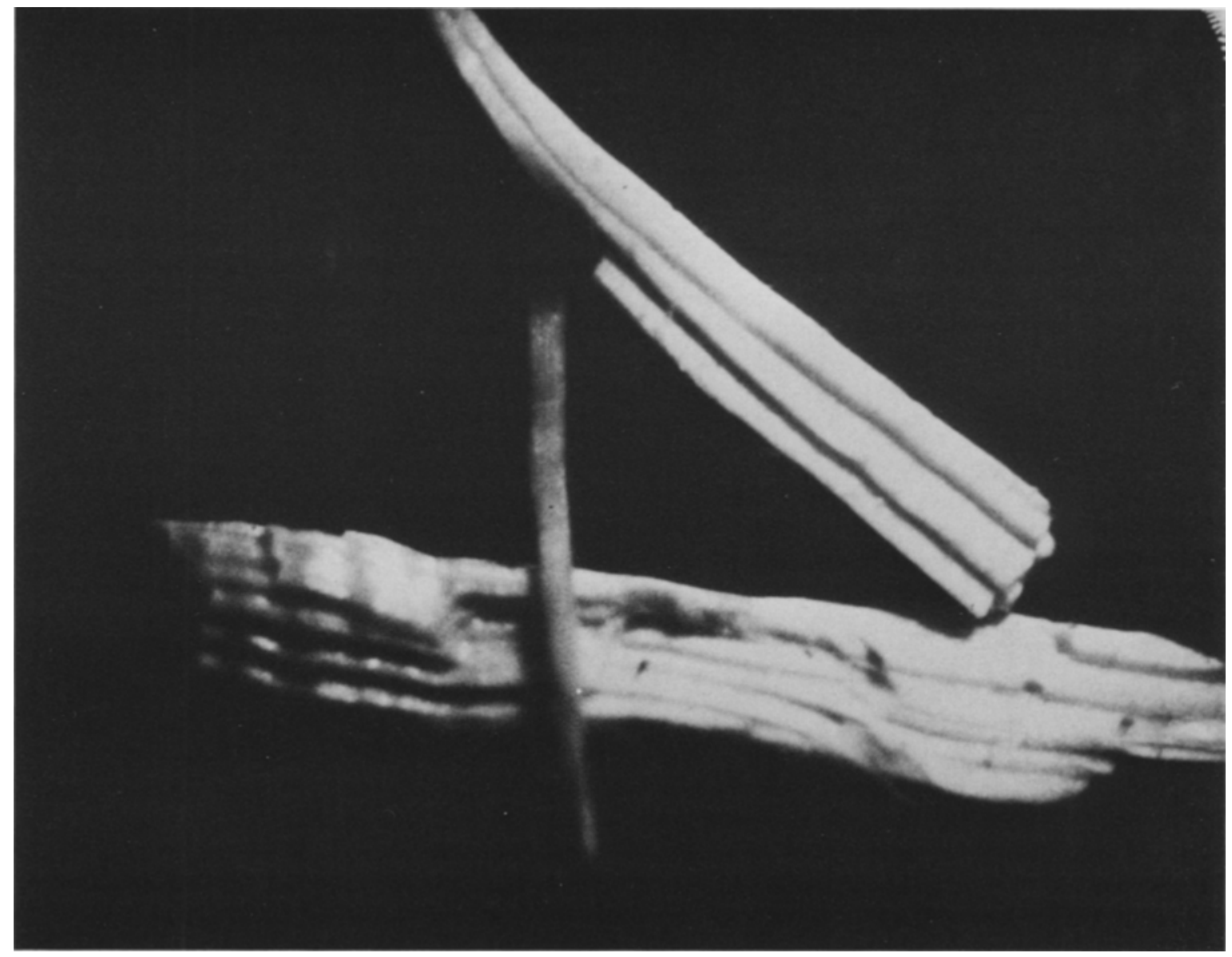

Figure 1. Frozen-dried portions of a biopsy from a normal human biceps muscle.

presents an analytical challenge of the kind that led Heinz Holter and KaI LinderstrøMLANG to develop their great quantitative histochemical methodology. We and others have therefore used their inspiration to find ways to determine the chemical composition of individual human and animal muscle fibers, the metabolite changes which occur with brief stimulation, and the enzyme changes which occur with long chronic stimulation $(3,8,11,12)$. This is a summary of some of our findings.

\section{METHODOLOGY}

The samples of individual fibers are obtained by the procedure described by EsSÉN et al. (3).
The muscle specimen is rapidly frozen. If labile metabolites are to be measured, the muscle is freeze-clamped in situ with metal tongs chilled to liquid $\mathrm{N}_{2}$ temperature. A piece, 5 or $10 \mathrm{~mm}$ long and not more than $1 \mathrm{~mm}$ thick, is freezedried at $-35^{\circ}$ to $-40^{\circ}$. This takes several days, rather than the few hours sufficient for microtome sections. From this piece, 2 to $5 \mathrm{~mm}$ lengths of individual fibers are obtained by teasing them apart with a razor blade knife or a fine needle (Figures 1 and 2). The individual fibers are stored under vacuum at $-25^{\circ}$ or below, and are exposed to room temperature and air only long enough to remove samples for a particular analysis.

For each assay, duplicate pieces are cut off 


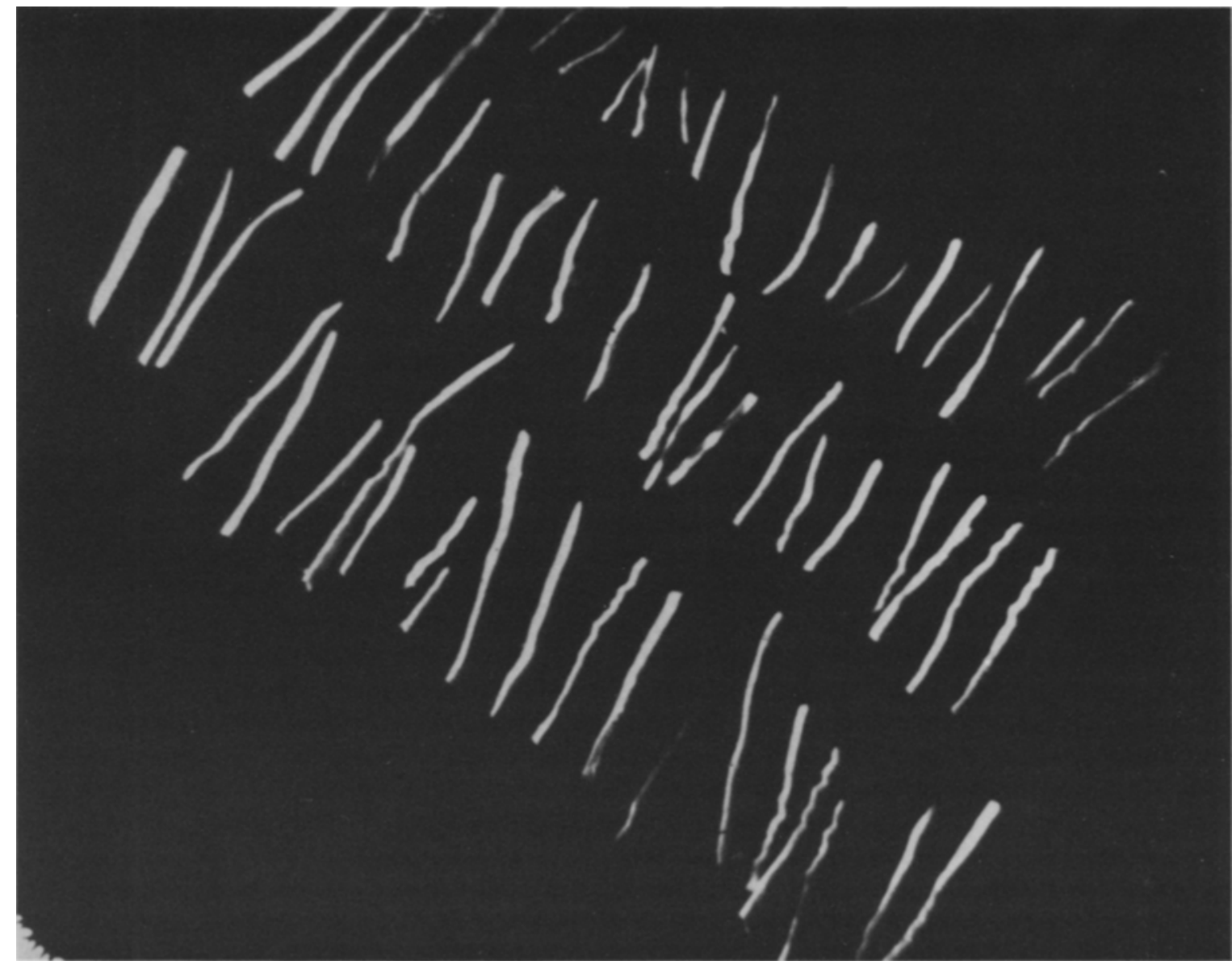

Figure 2. Segments of 57 different individual fibers obtained from the muscle samples of Figure 1.

one end leaving the rest for other measurements. Usually these pieces are in the 10 to $40 \mathrm{ng}$ (dry weight) range. Figure 3 shows the large numbers of suitable size samples that can be obtained from a 2 mm length of a single fiber.

The samples are weighed on a quartz fibers fishpole balance (9) and added to a 0.05 to 10 $\mu 1$ droplet of appropriate reagent under oil in a Teflon "oil-well" rack (9). Successive intermediate analytical steps are carried out in the oil well rack, but the final fluorescence measurement is made in a glass tubes at a volume of about $1 \mathrm{ml}$.

For measuring metabolites, and all but the most active enzymes, it is necessary to amplify sensitivity by "enzymatic cycling" (9). The ove- rall analytical process is illustrated by the following example for fructose bisphosphate.

Step 1. The sample containing 0.5 to $5 \mathrm{fmol}$ of fructose bisphosphate, is added to $\mathrm{HCl}(0.1$ $\mu \mathrm{l})$ and heated to destroy enzymes (i.e. the oil well-rack is heated).

Step 2. The sample is heated again after adding excess $\mathrm{NaOH}(0.05 \mu \mathrm{l})$ to destroy triose phosphates.

Step 3. The fructose biphosphate is converted to 3-phosphoglycerate with $\mathrm{NAD}^{+}$, arsenate and the necessary enzymes $(0.1 \mu \mathrm{l}$ of reagent) forming two equivalents of NADH.

Step 4. Excess $\mathrm{NAD}^{+}$is destroyed with $\mathrm{NaOH}$ $(0.1 \mu \mathrm{l})$ and heat. 


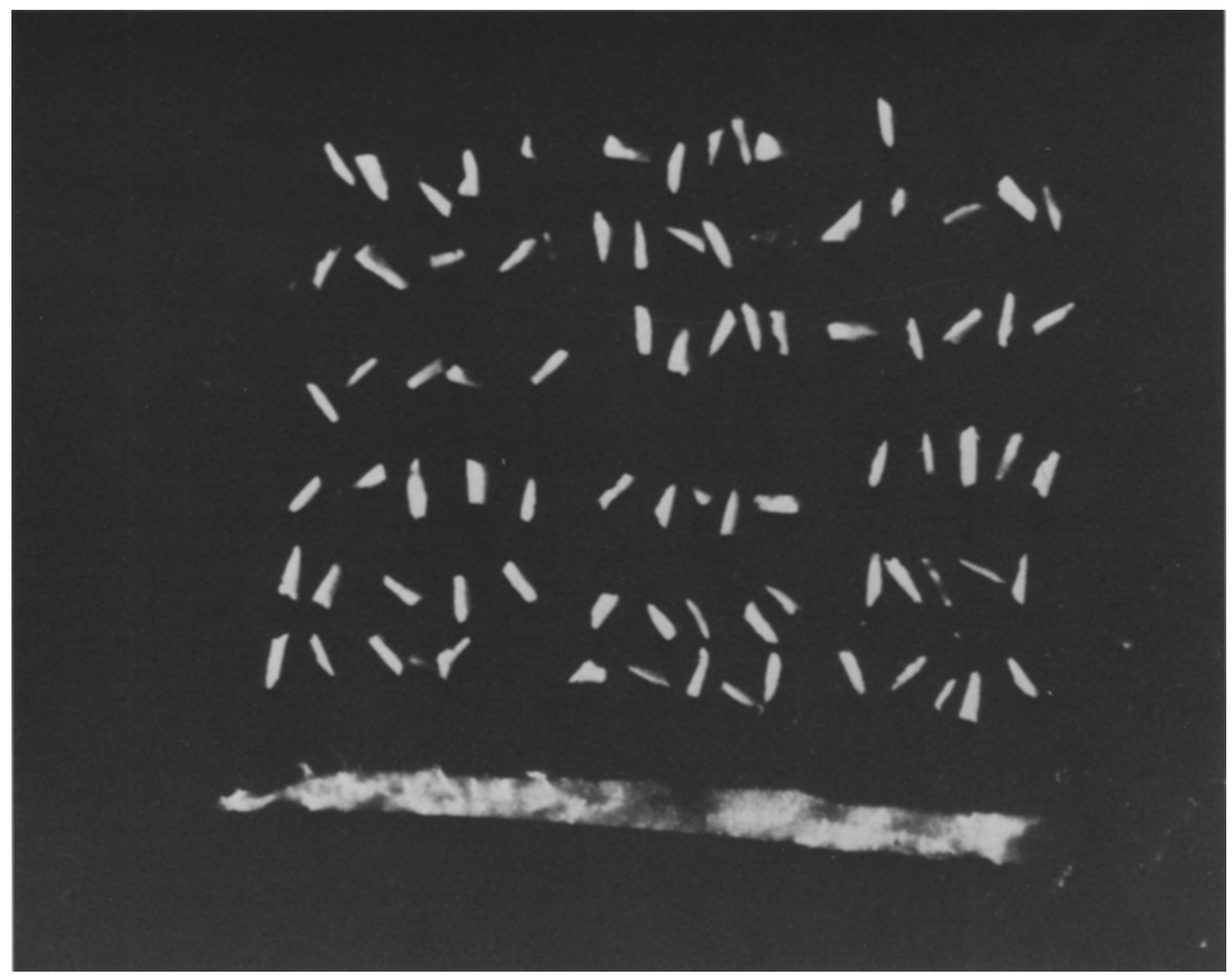

Figure 3. One of the fiber segments of Figure 2 cut into 86 pieces of convenient size for assay, together with an uncut adjacent segment from Figure 2.

Step 5. $5 \mu$ l of NAD cycling reagent (7) are added and incubated overnight to produce 150.000 moles of malate per mole of NADH:

acetaldehyde

ethanol

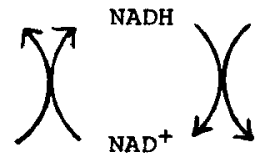

malate of NADH, which is now measured by its fluorescence:

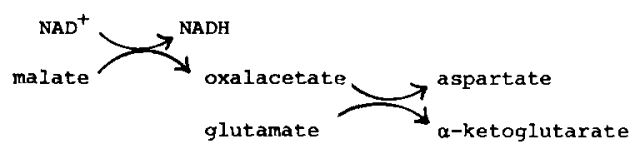

The malate oxidation is pulled to completion by the transaminase reaction. Note that the $\mathrm{NADH}$ produced in Step 3 has literally been amplified 150.000 fold (ignoring the portion of the sample left behind in the oil well at Step 6). 
O.H. LowRY: The heterogeneity of muscle

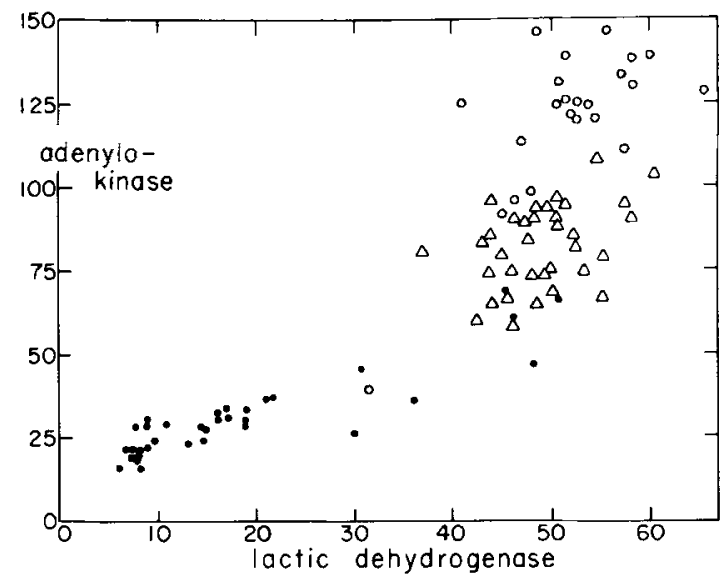

Figure 4. Adenylokinase and lactic dehydrogenase on 89 fibers from the same muscle. The numbers indicate activity as mol $\mathrm{kg}^{-1}$ (dry weight $\mathrm{h}^{-1}$ ). The symbols indicate the range of $\beta$-hydroxyacyl-CoA dehydrogenase activity for the same fibers in the same units: $\mathrm{C}$, $\ll 1.60 ; \mathrm{D}, 1.61$ to $2.75 ; \mathrm{V}, \gg 2.75$. Reprinted with permission from (8).

\section{RESULTS}

\subsection{Normal human fibers}

The extreme heterogeneity among skeletal muscle fibers is illustrated in Figure 4. This is a plot of 89 fibers from a biopsy of a normal human biceps muscle. Each fiber was assayed in duplicate for lactate dehydrogenase (LDH) and adenylokinase (8). The values spread, in each case, over a 10-fold range in an almost continuous spectrum, except for a distinct break in the middle. The lower group are Type I fibers the upper group Type IIA and IIB (see below).

These fibers were also analyzed for an enzyme of oxidative metabolism, $\beta$-hydroxyacyl $\mathrm{CoA}$ dehydrogenase. As shown, this enzyme was inversely related to $\mathrm{LDH}$ and adenylokinase (see Figure legend).

These data are typical of the distribution of other enzymes of oxidative and glycogenolytic metabolism among human muscle fibers, although the proportions between enzymes vary substantially. Thus in biceps muscles of five normal males (8), the range of citrate synthase levels was distinctly less than that of $\beta$-hydroxyacyl CoA dehydrogenase. Similarly the range of activities for glycogen phosphorylase was much less than for LDH.

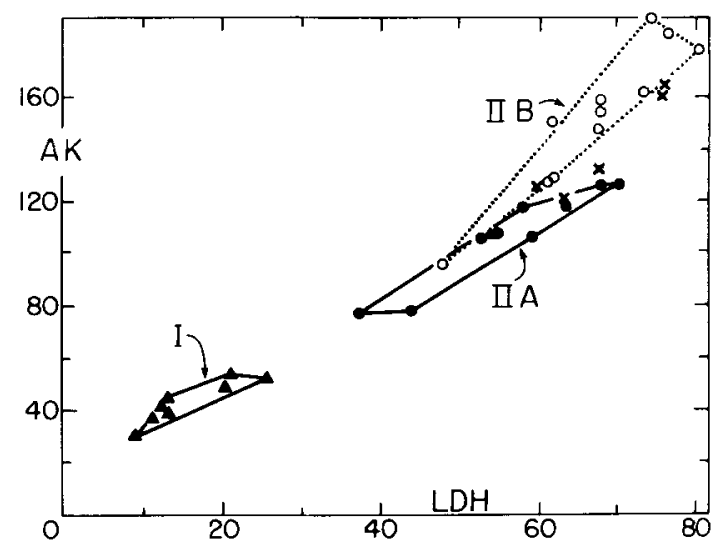

Figure 5. Lactate dehydrogenase (LDH) and adenylokinase (AK) activity of 33 individual human fibers. They are assigned to the types shown on the basis of ATPase staining. The fibers are from the vastus lateralis muscle of a 26 year old male volunteer. The staining of fibers marked " $X$ " was intermediate to that of IIA and IIB types. Activities are expressed as in Figure 4. Reprinted with permission from (6).

\subsection{Determination of fiber types.}

Fiber types have usually been determined on the basis of the "myosin ATPase" staining method developed by PADYKULA and HERMAN (10) and further refined by BROOKE and KAISER (1), Guth and SamaHa (4) and others. The method depends on differential staining of muscle sections pretreated at different $\mathrm{pH}$ 's.

It seemed important to compare type designations made on this basis with the types shown by quantitative enzymes assays. It was difficult to do this with fibers isolated as described above. Instead transverse microtome sections were cut at $14 \mu \mathrm{m}$ from intact muscle. A few sections were air dried and stained for myosin ATPase; the rest were freeze-dried. Photomicrographs of the stained sections were used as a guide to select fibers of the different ATPase types from the freeze-dried sections. The cross sections of the fibers selected were dissected out, cut in half to provide duplicate assays, weighedand analyzed (6).

Figure 5 is typical result for a human muscle. The fibers designated Type I and Type II by the ATPase stain are clearly separated on the LDH-adenylokinase grid, but there is some overlap among the Type IIA and IIB subtypes. 


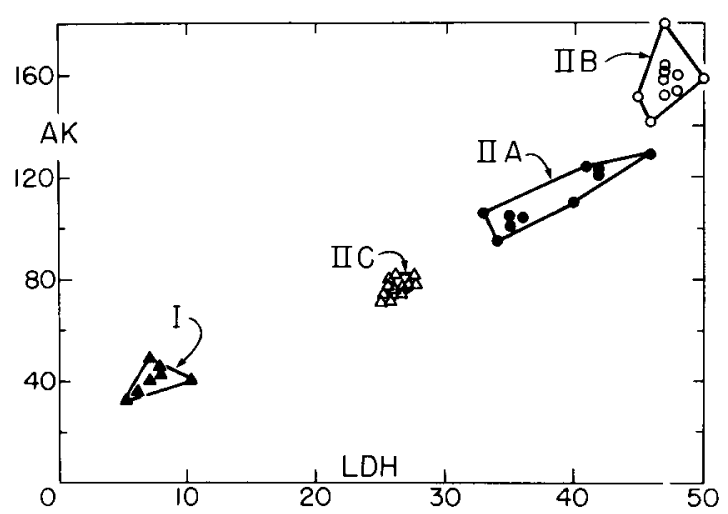

Figure 6. Adenylokinase and LDH levels in fibers from a vastus lateralis muscle found to have numerous Type IIC fibers. The muscle was from a 36 years old normal male volunteer. Activities are expressed as in Figure 4. Reprinted with permission from (6).

Figure 6 illustrates the results for an individual with an unusual number of ATPase Type "IIC" fibers, a type not previously categorized as to metabolic enzyme characteristics. As shown, they fall between the Type I and IIA fibers on the enzyme grid.

\subsection{Effects of training and detraining}

Major changes in muscle metabolic enzymes can be induced by excercise or other types of chronic stimulation. Figure 7 shows citrate synthase and $\mathrm{LDH}$ values in the vastus lateralis muscle from an untrained and a highly trained man, and from the same trained man after training had been stopped for 7 weeks (2). The fiber typing is based on LDH and adenylokinase levels (not shown), with no attempt to distinguish between IIA and IIB types. Note the complete overlap in citrate synthase values of the type I and II fibers for the trained individual, and that although with detraining citrate synthase decreases, particularly in Type II fibers, values for many fibers were still found in the trained range. However, after the 7 weeks of detraining the levels of this enzyme in Type II fibers had fallen in only a few cases into the range of the untrained person. In contrast to citrate synthase, $\mathrm{LDH}$ activities had risen during the detraining period to at least the untrained range.

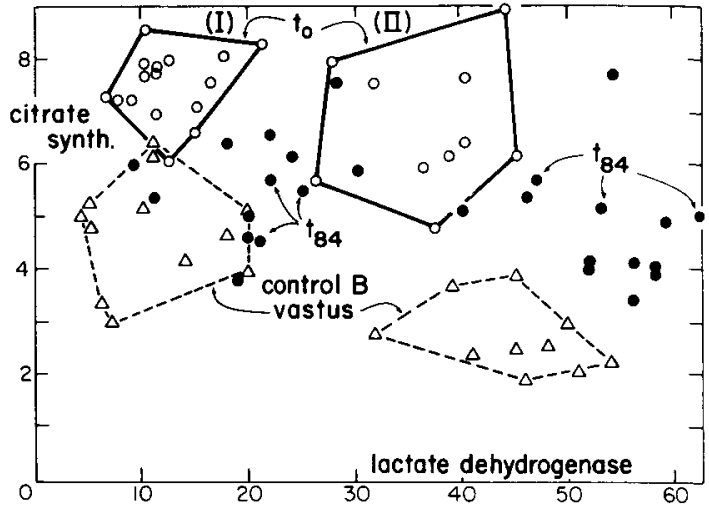

Figure 7. Citrate synthase and LDH in fibers from the vastus lateralis muscle of cyclist during training $\left(t_{0}\right)$ and after detraining for 84 days $\left(t_{84}\right)$, together with vastus fibers from an untrained person ("Control B"). Activities expressed as in Figure 4. Reprinted with permission from (2).

\subsection{Effects of chronic motor nerve stimulation}

Much greater changes than those induced by training can be produced by chronic stimulation in experimental animals. Figures 8 and 9 give examples from a study of changes in a fast twitch rabbit muscle stimulated at a slow frequency for up to 10 weeks, night and day. The initial fiber composition was on the average about $70 \%$ IIB, $25 \%$ IIA and $5 \%$ or less Type I (based on myosin ATPase stains). In the example shown (Figure 8), LDH levels varied only about $50 \%$ but malate dehydrogenase (MDH) activities spread over a 10-fold range. After 2 weeks, all the low $\mathrm{MDH}$ values had disappeared without increase in LDH. Most of the fibers now gave ATPase stains typical of Type IIA and IIC.

By 8 weeks, MDH had not risen much more but LDH and fallen to an average of $20 \%$ of the inital values. At this time, most of the fibers stained like Type I fibers. However, the levels of LDH and especially those of MDH differed greatly from the levels in the control soleus muscle (Figure 8) which contains almost exclusively Type I fibers.

\subsection{Effects of stimulation on metabolite levels}

Skeletal muscle at rest has a very low metabolite rate. However, with maximal stimulation, energy expenditure can increase several 


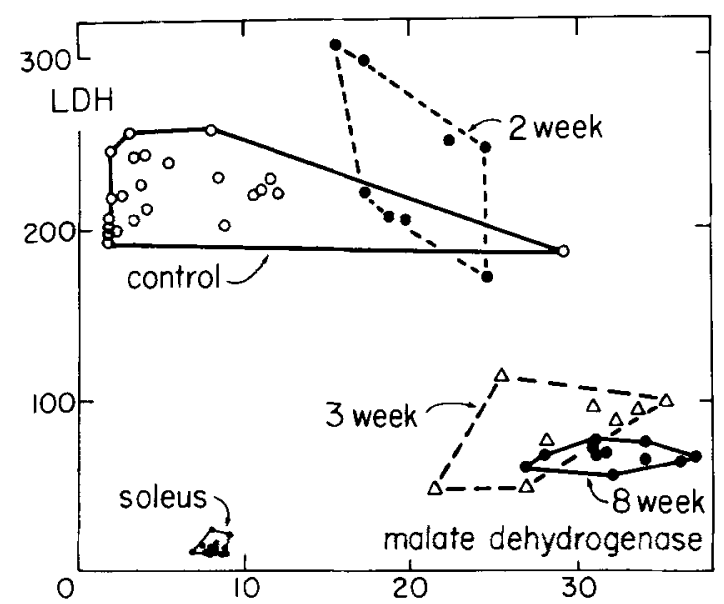

Figure 8. Malate dehydrogenase (MDH) and LDH in fibers from a rabbit anterior tibialis muscle that had been stimulated continuously for the time shown at $10 \mathrm{~Hz}$ with subcutaneous device attached to electrodes around, but not touching, the peroneal nerve. From unpublished data obtained by J. HENRIKSSON. S. Salmons, P.N. Nemeth. M.M.Y. Chi, J. PARk, C.S. HinTZ. P.N. NEMETH and O.H. LOWRY. Activities are recorded as in Figure 4.

hundred-fold to reach extremely high levels. This forces the energy-converting mechanisms into high gear with dramatic changes in high energy phosphates and levels of metabolites along the pathways of energy conversion. Given the big differences in metabolic enzymes among the fibers of any given muscle, it is not surprising that there should be major interfiber differences in metabolite responses as well.

Figure 9 shows such differences for ATP and $P$-creatine among fibers of two rat muscles, one which contained mainly IIA and IIB fibers and another which contained almost entirely Type I fibers (5). During a 1 minute stimulation, P-creatine fell $70 \%$ in IIB fibers and had almost disappeared by 15 minutes. In contrast, P-creatine only fell $8 \%$ in Type IIA fiber and $32 \%$ in Type $I$ during the first minute and had actually recovered somewhat by 15 minutes (not shown). Small but significant increases were seen in all fiber types in the first minute and are attributed to the shift in $\mathrm{pH}$ caused by lactic acid formation. By 15 minutes, however, ATP had fallen drastically in IIB fibers (Figure 9) without essential change in either Type IIA or Type I fibers (not shown). These results clearly

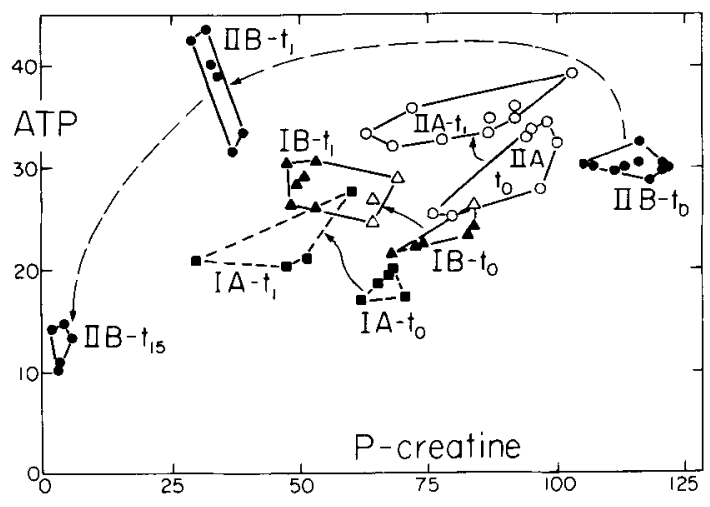

Figure 9. Correlation between ATP and P-creatine in control fibers $\left(t_{0}\right)$ and fibers stimulated supramaximally for 1 minute $\left(t_{1}\right)$ or 15 minutes $\left(t_{15}\right)$ with 100 ms trains of $50 \mathrm{~Hz}$ delivered at a rate of $60 / \mathrm{min}$. Type I fibers at the lower end of the LDH scale are arbitrarily designated "IA", the rest are "IB". Type II fibers are from plantaris muscle and IA are from soleus. IB fibers are from both soleus (solid triangles) and plantaris (open triangles). Reprinted with permission from (5).

show the greater fatigue resistance of IIA and I fibers.

Figure 10 illustrates the different effects of the increased metabolite flux during stimulation on a metabolite of the citrate cycle, and on the terminal metabolite of glycogenolysis. Lactate increases were greatest in IIB fibers and least in Type I. Malate concentration was high to begin with in Type I fibers, and at all times was higher than in any other group.

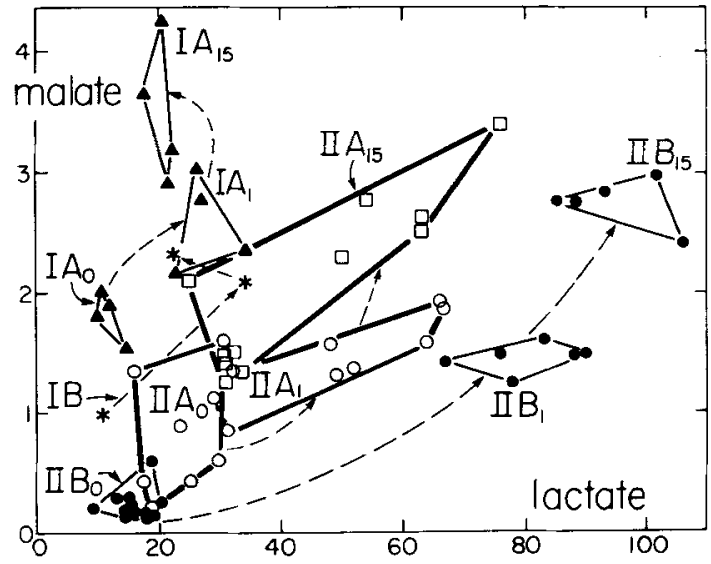

Figure 10. Relationships between malate and lactate in the same individual fibers shown in Figure 9. Individual IB fibers have been omitted to avoid confusion. Reprinted with permission from (5). 


\section{CONCLUSION}

Over the years many people have applied the direct approach of HOLTER and LANG to unravelling some of the complexities of a wide variety of organs. But in the case of muscle, there was a delay in applying this approach because people thought of muscle as an organ with little heterogeneity, at least in regard to the muscle cells themselves. As it turns out, and as this presentation underscores, muscle has proved to be a very complex organ indeed, and one particularly well suited to direct quantitative histochemical study. The combination of fiber heterogeneity, the extreme metabolite changes that can be produced by brief stimulation and the major changes in metabolic machinery that can be effected by chronic stimulation, provide a fascinating opportunity to study enzyme intergration and adaptive mechanisms and a most proper object for desciples of the Carlsberg Masters.

\section{ACKNOWLEDGEMENTS}

Supported in part by Research Center Grant from the Muscular Dystrophy Association of America and a grant from the National Institutes of Health (NS-08862).

\section{REFERENCES}

1. BroOKe, M.H. \& K.K. KaISER: Muscle fiber types: how many and what kind? Arch. Neurol. 23 369-379 (1970)

2. Chi, M.M.Y., C.S. Hintz, E.F. CoYle, W.H. MaRTIN III, J.L. IVY, P.M. NeMETH, J.O. HOLLOSZY \& O.H. LowRY: Effects of detraining on enzymes of energy metabolism in individual muscle fibers.

. Am. J. Physiol. 244 (Cell Physiol. 13), C276-C287 (1983)

3. EsSEn, B., E. JANSSON, J. Henriksson, A.W. TAY-
LOR \& B. SALTIN: Metabolic characteristics of fiber types in human skeletal muscle. Acta Physiol. Scand. 95, 153-165 (1975)

4. Guth, L. \& FJ. Samaha: Qualitative differences between actomyosin ATPase of slow and fast mammalian muscle. Exp. Neurol. 25, 138-163 (1969)

5. HINTZ,C.S., M.M.-Y.CHI.R.D. FELL.J.L. IVY. K.K KaISER, C.V. LOWRY \& H.O. LOWRY: Metabolite changes in individual rat muscle fibers during stimulation. Am. J. Physiol. 242 (Cell Physiol. 11) C218-C227 (1982)

6. Hintz, C.S. E.F. Coyle, K.K. Kaiser, M.M.Y. CHI \& O.H. LOWRY: Comparison of muscle fiber typing by qualitative enzyme assays and by myosin ATPase staining. J. Histochem. Cytochem. in press

7. Kato, T., S.J. Berger, J.A. Carter \& O.H. LOWRY: An enzymatic cycling method for nicotinamide-adenine dinucleotide with malic and alcohol dehydrogenases. Anal. Biochem. 53, 86-97 (1973)

8. LOWRY. C.V.. J.S. KimMEY, S. Felder. M.M.Y. Chi, K.K. Kaiser, P.N. Passonneau. K.A. Kirk \& O.H. LowRY: Enzyme patterns in single human muscle fibers. J. Biol. Chem. 253, 8269-8277 (1978)

9. Lowry O.H \& J.V. Passonneau: A Flexible System of Enzymatic Analysis. New York: Academic (1972)

10. Padykula. H.A. \& E. Herman: The specificity of the histochemical method of adenosine triphosphatase. J. Histochem. Cytochem. 3, 170-195 (1955)

11. Rehunen, S. \& M. HaRKonen: High-energy phosphate compounds in slow twitch and fast-twitch muscle fibers. Scand. J. Clin. Lab. Invest. 40, 45-53 (1980)

12. Spamer, C. \& D. Pette: Activity patterns of phoshofructokinase, glyceraldehydephosphate dehydrogenase, lactate dehydrogenase and malate dehydrogenase in microdissected fast and slow fibers from rabbit psoas and soleus muscle. Histochemistry $52,201-216$ (1977) 